\title{
El Aprendizaje Creativo y el Cerebro. Rescatar el Concepto de “Aprehender"
}

\section{The Creative Learning and the Brain. To Rescue the Concept of “Apprehending”}

\author{
Francisco Menchén Bellón *
}

\begin{abstract}
El reto de la educación en las próximas décadas no será, simplemente, el dominio del conocimiento, sino el poder del aprendizaje creativo. La era del aprendizaje repetitivo pertenece al pasado. En el siglo XXI brillará el aprendizaje creativo, que será la herramienta principal del aula. Los alumnos del futuro tendrán que aprehender de forma holística, permanente y creativa. Asociar educación a escuela es un pensamiento propio de la revolución industrial y no de la revolución de la creatividad. Todo cuanto conocemos y sabemos no es más que una creación de la mente humana, fruto del aprendizaje creativo. El hecho de asumir una nueva manera de entender el aprendizaje, lleva consigo la modificación del patrón de comportamiento o los pertinentes algoritmos. Todo depende del sentido que le demos a lo que hacemos. Para implementar en el aula el aprendizaje creativo el docente debe contemplar dos factores: rescatar el concepto de aprehender, y conocer el funcionamiento del cerebro a la hora de enseñar. En consecuencia, el docente debe respetar el ritmo de aprendizaje creativo que tiene cada alumno y además contemplar que, para crear conocimiento es necesario conocer el proceso cerebral que interviene. Para gestionar este cambio, es imprescindible salirse de la zona de confort e inspirar a los alumnos a desarrollar habilidades nuevas y despertar pasión por aprehender.
\end{abstract}

Descriptores: Creatividad; Aprendizaje; Conocimiento; Cerebro; Aula.

The challenge of education in the coming decades will not be the mastery of knowledge, but the power of creative learning. The era of repetitive learning belongs to the past. In the 21 st century creative learning will shine, and will be the main tool in the classroom. Students of the future will have to apprehend in a holistic, permanent and creative way. Linking education to school is a thought of the industrial revolution and not of the revolution of creativity. Everything we know is nothing more than a creation of the human mind, the fruit of creative learning. Assuming a new way to understand the learning process, carries with it the modification pattern of the customary behavior or the pertinent algorithms. It all depends on the sense we give to what we do. To implement creative learning in the classroom, the teacher should contemplate two factors: to rescue the concept of apprehending, and to know the functioning of the brain when it comes to teaching. Accordingly, the teacher must respect the creativity learning pace of each student; In addition, the teacher must consider that to create knowledge is necessary to know the brain process involved. To manage this change, it is essential to get out of the comfort zone and inspire students to develop new skills and arouse a passion for apprehending.

Keywords: Creativity; Learning; Knowledge; Brain; Classrooms.

*Contacto: fcomenchen@yahoo.com

ISSN: 2254-3139

www.rinace.net/riejs/

revistas.uam.es/riejs
Recibido: $\quad 10$ de agosto 2017

$1^{\text {a }}$ Evaluación: 2 de enero 2018

$2^{\text {a }}$ Evaluación: 16 de febrero 2018

Aceptado: $\quad 25$ de mayo 2018 


\section{Introducción}

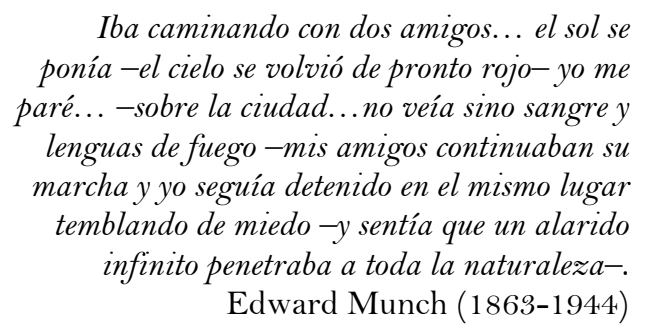

¿Cómo ir más allá del aprendizaje clásico?

Escribiendo este texto me ha venido a la memoria el célebre cuadro "El grito" que Edward Munch pintó con gran expresividad en 1893. El pintor noruego tenía 29 años y se enfrentó cara a cara con la realidad. No hay escape: la revelación o mejor dicho la revolución está delante del célebre artista. Esta visión tan gráfica de un genio es sobrecogedora, hoy día también, pues toca en el ser humano algo profundo, que debe servir para dar sentido al trabajo cotidiano.

La experiencia que produce esta impresionante imagen obligará a los docentes a ver la realidad con ojos diferentes y percibir que el centro de gravedad de un aula debe cambiar. Seguro que tendremos varias alternativas, pero mi propuesta es simple: implementar el aprendizaje creativo en el aula y conocer cómo funciona el cerebro por parte del docente; es una nueva perspectiva que conectará con nosotros mismos y con nuestros talentos. Es la única posibilidad que queda.

Las nuevas generaciones de alumnos tendrán diversas formas de aprender, y esto les posibilitará elegir nuevas experiencias, aprendizajes y conocimientos. La modalidad de aprendizaje que cada uno tiene será muy peculiar: procesarán y generarán la información de manera muy singular; unos sujetos serán capaces de interiorizar y vivenciar lo aprendido, mientras otros se conformarán con experimentar meras sensaciones sensoriales.

La realidad es que muchos centros educativos siguen operando muy por debajo de las posibilidades que hoy día atesoran los alumnos. Pocos alcanzan la excelencia en el aprendizaje creativo. Con ello, no quiero decir que se impida el aprendizaje repetitivo, sino que, hemos de ir más allá y empezar a vivir en una sociedad que promueva el más alto nivel de aprendizaje, de tal forma que, cambie el punto de vista y permita el crecimiento interior. Asociar educación a escuela es un pensamiento propio de la revolución industrial y no de la revolución de la creatividad. Esta novedosa visión que propongo proporcionará un enfoque, completamente, desconocido, pero ya ha empezado a ser el foco de atención de la ciencia, como también debe extenderse a la dimensión educativa del proceso de aprendizaje. En el siglo XXI no se puede seguir sosteniendo la visión reduccionista. Las mentes conectadas están liderando la revolución creativa.

Venimos a este mundo a ser feliz, amar, disfrutar y compartir todas las situaciones de la vida, pero también hemos llegado a este planeta para aprender y enseñar La vida es un lienzo en blanco y cada momento con nuestras creencias, pensamientos, decisiones y actitudes pintamos el paisaje en el que queremos vivir. Todo cuanto conocemos y sabemos no es más que una creación de la mente humana, fruto del aprendizaje creativo. No hay nada predeterminado de antemano, sino cada uno crea su realidad mediante sus elecciones personales a lo largo de su vida, procurando siempre que sea para un beneficio propio o 
colectivo. John Dewey (1859-1952), pedagogo americano ilustre, puso en la órbita educativa el modelo learning by doing (aprender haciendo), que, en su evolución natural, hoy se transformaría en "aprender creando". El ser humano tiene que reinventarse cada día.

Hay una famosa sentencia que dice: "escucho y olvido, veo y recuerdo, hago y comprendo" que nos conduce a pensar que el empleo de la lógica, la razón y los datos no son suficientes. Para alcanzar el aprendizaje creativo se requiere acción, entusiasmo y pasión. El progreso de un país depende del nivel de aprendizaje de su población. En el pasado el aprendizaje clásico marcó la época, generando gran beneficio y bienestar. Pero ahora, es necesario un nuevo enfoque, ya que vivimos en una sociedad en continuos cambios, que innova y se desarrolla a mayor velocidad, por lo que se requiere una redefinición del modelo de aprendizaje y nuevas estrategias.

El contenido de nuestra aportación se inicia con la justificación de la necesidad imperiosa de promover e implementar el aprendizaje creativo, que dé respuestas a las necesidades de una sociedad compleja que avanza desnortada por la incertidumbre que plantea el futuro. Para tal fin hemos rescatado el concepto aprehender por considerar que es clave para emprender una nueva forma de crear el conocimiento.

También reflejamos la importancia que tiene para el docente conocer cómo funciona el cerebro y las huellas que dejan las experiencias de aprendizaje en los alumnos. En consecuencia, abordaremos el impacto que esto supone en el campo de la educación.

\section{Objetivos}

Afortunadamente, en la actualidad se están multiplicando las investigaciones en este campo, y todas ellas vienen a confirmar que un entorno estimulante fortalece las conexiones entre neuronas, la materia prima del aprendizaje creativo, mientras que un ambiente tenso hace que las conexiones sinápticas desaparezcan. Por todo ello, esta contribución se concreta en los objetivos siguientes:

- Implementar el aprendizaje creativo a las nuevas generaciones de alumnos, y para ello, se requiere rescatar el concepto de "aprehender".

- Conocer el funcionamiento del cerebro es imprescindible para el docente, si pretende que sus alumnos sean capaces de dominar el aprendizaje creativo.

La experiencia nos dice que debemos olvidar lo aprendido y desprendernos de antiguos hábitos, que en su tiempo fueron útiles, pero que ahora han perdido vigencia. Es necesario vaciarse de lo antiguo e incorporar energía actualizada. En definitiva, es la conciencia del sujeto responsable de adaptarse a las nuevas circunstancias, trasmitiendo al cerebro las emociones y sentimientos apropiados para influir en el proceso del aprendizaje creativo. Por consiguiente, nuestra propuesta se sintetiza como sigue: para implementar el aprendizaje creativo en el aula, el docente debe contemplar dos estrategias: (i) Rescatar el concepto de "aprehender" con el objeto de darle más sentido y profundidad a cualquier tipo de aprendizaje. (ii) Para que el proceso de enseñanza-aprendizaje sea más efectivo es fundamental conocer el funcionamiento del cerebro. 


\section{Qué entendemos por aprendizaje creativo}

El énfasis de la educación no debería estar tanto en lo que deben aprender los estudiantes, sino en qué podrán hacer con lo que aprendan.

(Torrance, 1976)

$¿$ ¿Conoces la esencia del aprendizaje creativo?

Entendemos por aprendizaje creativo, el arte de saber emplear la energía necesaria para conseguir una nueva conexión de las neuronas, que ayuden a reconstruir, reelaborar y ampliar los conocimientos de forma consciente. Esta conexión neuronal debe ir acompañada de una emoción, capaz de producir vibraciones sincronizadas, con frecuencias más o menos elevadas.

El aprendizaje creativo se genera a partir del conocimiento de un objeto, en función de los órganos sensoriales, luego estas percepciones y sensaciones se han de organizar y darle un sentido, pasando posteriormente a confrontar y comparar con la realidad para identificar su vigencia. Es de esta forma como se genera el conocimiento, que con el paso del tiempo cambia, se modifica o perfecciona; es decir, evoluciona. El conocimiento no es estático, cambia continuamente, gracias a las nuevas investigaciones de las ciencias.

El aprendizaje creativo es la mejor forma para construir el conocimiento, siendo imprescindible dominar la materia en cuestión, contar con un pensamiento reflexivo y utilizar una metodología adecuada. Desde tiempos antiguos el hombre ha tratado de explicar todo tipo de fenómenos, creando las herramientas idóneas para adaptarse mejor a su medio ambiente, conocer y transformar la realidad. Gracias a esta modalidad de aprendizaje se han descubierto y creado los viajes espaciales, internet, y drones, entre otros muchos inventos.

Existen infinitas posibilidades para que el docente promueva el aprendizaje creativo, pero en muchas ocasiones está limitado por las creencias y la estrechez mental. Abrir la mente a estas nuevas posibilidades puede derivarse en potenciar un alumno multi-talento, capaz de tomar conciencia de las infinitas capacidades de que dispone. Para conseguir el aprendizaje creativo es imprescindible detectar las señales intuitivas que fluyen por nuestro interior. Para que cristalice un aprendizaje creativo hace falta un impulso mental, más allá del simple deseo, un empeño consciente, que supone una poderosa mezcla entre la conciencia y la voluntad. Sabemos que los pensamientos subconscientes, habitualmente, pueblan nuestra conciencia, y son los que nos pautan la vida, e influyen en el aprendizaje. Los alumnos son los únicos responsable de aprehender o no, dependiendo de las elecciones personales que hagan.

Para que el aprendizaje creativo surja debemos conectar de manera consciente con el inconsciente, reordenando el caos creado por la conciencia cuántica. Por esta razón, el docente deberá prestar atención a la mente subconsciente, mediante la respiración profunda. Lo que pensamos, sentimos e imaginamos debe formar parte del proceso creador de la realidad de cada día. El objetivo final del aprendizaje creativo es que el alumno sea capaz de activar el conocimiento adquirido, y generar buenas preguntas, cuando tenga que actuar en otras situaciones. Se ha demostrado que un conocimiento significativo no se aprende leyendo textos, sino requiere la aplicación de procesos que tengan en cuenta las ideas previas y el modo en que se progresa en dicho conocimiento. Lev S. Vygotski (18961934), psicólogo ruso, lo expresa con claridad: "El conocimiento que no proviene de la 
experiencia no es realmente un saber". Cuando un alumno no aprende puede ser, porque su conciencia no encuentra sentido a la enseñanza que recibe, o también por una incapacidad de maniobrar la mente. Si aplicamos la teoría junguiana, podemos decir que, se basa en no tener asumido la expresión "soy capaz". Cuando el docente ayuda al sujeto a borrar el arquetipo "no puedo" que le impide aprehender, y en su lugar interiorice "si puedo", reordenará su mente y forjará una nueva realidad, que cambiará todos los mensajes que circulan por el cerebro, así como los pertinentes nutrientes químicos que alimentarán a las células.

Recomiendo que, el maestro o profesor seleccione, cuidadosamente, el lenguaje, las ideas y las emociones, porque la vibración es poderosa y modela día a día, de forma lenta pero profunda y puede conducir al éxito o bien al fracaso, pues las vibraciones o energía electromagnética que produce una persona hablan sobre su estado interior. Para que exista aprendizaje creativo es esencial que el alumno y el docente estén en sintonía. Esto ocurre, cuando ambas mentes resuenan, sincronizan y mantienen el equilibrio interior necesario para complementarse. Podemos decir que el maestro y el discípulo están confabulados y están fluyendo en la misma onda. Si no cambias puedes quedar atrapado por el arquetipo "no puedo", con el consiguiente deterioro. Pero si cambias, se ejerce una influencia consciente sobre el entorno inmediato, invisible y subliminal. "Cambiar de arquetipo es un proceso creativo en el que la imaginación juega un papel fundamental” (Saint Aymour, 2015, p. 142).

Hay que romper las cadenas del determinismo que nosotros mismos hemos creado. Nuestro cerebro racional nos boicotea algunas veces. Cuando la conciencia se posa en una nueva idea surge la chispa que desencadena nuevos acontecimientos, generados por el deseo de aprehender, debido al vínculo que se establece entre el pensamiento y el sistema creativo. "El pensamiento determina la acción, la acción determina el pensamiento" (Principio del budismo). El hecho de asumir una nueva relación en la forma de entender el aprendizaje, hace posible la modificación del patrón de comportamiento o los pertinentes algoritmos y, en ese momento se forma una nueva realidad. Todo depende del sentido que le demos a lo que hacemos. El éxito consiste en entrenar las sincronicidades, enfocando con la debida intensidad lo que deseas que ocurra. Es decir, se trata de crear un puente entre el pensamiento consciente, y el inconsciente, a través de la energía.

\section{Rescatar el concepto de Aprehender}

Quien mira hacia fuera, sueña; quien mira hacia dentro, despierta.

(Carl Jung, 1875-1961)

¿Qué diferencia hay entre aprehender y aprender?

Aprender y aprehender suenan casi iguales y tienen la misma etimología. Ambas palabras proceden del latín aprehendere. Con el paso del tiempo aprender perdió la "h" y se usó para incorporar un nuevo conocimiento a la estructura cognitiva y aprehender preservó la " $\mathrm{h}$ " originaria que se traduce por "atrapar". Como rasgos determinantes se puede decir que, cuando un alumno interactúa con el entorno aprehende y cuando estudia aprende; asimismo, los conocimientos adquiridos a través de aprender se pueden olvidar con el tiempo ya que el sujeto no los internaliza, mientras, con aprehender los conocimientos jamás se olvidan. Aprender palabras es muy distintos a aprehender conceptos. 
Desde otra perspectiva, se puede decir que la mayoría de los alumnos son puros consumidores de conocimientos que poseen los otros, es el caso del aprendizaje habitual, que consiste en reproducir información -aprendizaje memorístico-; sin embargo, muy pocos estudiantes producen, construyen o fabrican sus propios conocimientos, que se identifican con aprehender, donde la información debe ser asimilada e integrada en los conocimientos anteriores, estableciendo nuevas relaciones -aprendizaje significativo-. Hemos rescatado el concepto de aprehender con la intención de que el alumno atrape el conocimiento desde distintas perspectivas, haciéndolo propio de modo duradero, y no solamente para pasar de curso; se encuentra vinculado al aprendizaje de una actividad o materia, pues se refiere a conseguir comprender algo. Este término es usado también como sinónimo de asir, agarrar, detener, aprisionar, entre otras acepciones.

Todo alumno tiene que tener la mentalidad de aprehender y crear algo nuevo cada día. El verdadero aprendizaje llega al corazón. A través de este proceso se puede contemplar que, todo lo que se hace puede hacerse mejor, bien interactuando o innovando. Es decir, es un sistema que nos capacita para hacer algo que antes no podíamos hacer, a la vez que, amplía la capacidad para crear y formar parte del proceso generativo de la vida. Tratando de actualizar el término "aprehender" he sintonizado con sorpresa con uno de mis gurús: Wilber (2016, p. 137) quien destaca el significado de "tocar", de tal forma que si tocas y abrazas un objeto lo "aprehendes". Explica que esta aprehensión (o conexión) también lo "trasciende" porque añade un poco de novedad creativa.

El aprendizaje creativo debe ser un proceso de construcción que supone analizar, comparar, criticar y finalmente, aplicar a casos concretos y descubrir que funciona. Estaríamos hablando del homo fabers En esta modalidad de aprendizaje el alumno construye un nuevo nivel de conocimiento a través de tocar, coger, prender y capturar aquello que tiene sentido para él. Se trata de aprender mejor, con sentido, con eficiencia, con pasión, con ilusión. Todo ello depende de cómo se perciba y se procese el mundo circundante. Este tipo de aprendizaje es distinto del aprendizaje mecánico, basado únicamente en la memoria, cuya única finalidad es aprobar exámenes.

Aprehender el conocimiento esencial es tan importante como crear equilibrio entre pensar, sentir y actuar. El ser humano tiene la disposición de aprehender solo aquello que le encuentra sentido o lógica, en este caso, lo percibe conscientemente y lo agarra y conecta para hacerlo suyo. El conocimiento forma parte de un todo, no se encuentra aislado. Motivar al alumno, de forma intrínseca, es la clave para aprehender y conseguir el aprendizaje creativo, ya que despierta la curiosidad intelectual, donde un nuevo pensamiento sirve de catalizador, a partir del cual surgen nuevas ideas y, por lo tanto, aparecerá los hilos que componen la urdimbre del tejido creativo. La motivación intrínseca es mucho más fuerte que las recompensas extrínsecas.

El alumno que tiene dificultades para aprehender puede ser por tener un nivel de aprendizaje bajo o muy bajo, o por el uso de un patrón neuronal mal estructurado. Si queremos alcanzar un nivel excelente hay que cambiar ese modelo y reprogramar la mente mediante un acto de imaginación creativa, mediante la focalización consciente de la idea que deseas conseguir. Si el ser humano tiene el poder de transformar su vida a través de la conciencia, cualquier alumno puede activar este poder interior e influir en el entorno inmediato, con el objeto de conseguir el aprendizaje creativo. Esta experiencia te convierte en un sujeto distinto ya que ha cambiado el modo de actuar. 
Los profesores deben saber que un alumno confiado, alegre y lleno de amor responderá positivamente y aprenderá con facilidad. Pero un alumno nervioso o tenso, se comportará mal. La autoestima y la confianza irradian energía y luz para entusiasmarse con su trabajo. La obligación o culpabilidad no son buenas razones para aprender, pues expresan un sentimiento de impotencia.

\section{Conocer el funcionamiento del cerebro}

Todo hombre puede ser, si se lo propone, escultor de su propio cerebro. (Ramón y Cajal, 2006)

¿Por qué es importante para el docente conocer los secretos del neuroaprendizaje?

El docente debe saber que, cuando el cerebro se nutre, adecuadamente, los mensajeros químicos que circulan por su interior favorecen el desarrollo armónico de las células y los órganos vitales. El alumno que domina el aprendizaje creativo, vive experiencias enriquecedoras, tanto intelectuales, como emocionales y sociales; ello le permite conectar con la naturaleza y la energía cósmica. El cerebro es un universo fascinante.

El cerebro humano es la estructura más compleja de la creación, que contiene más neuronas, que estrellas existentes en nuestra galaxia. Esta "máquina" casi perfecta dicta toda nuestra actividad intelectual, emocional y creativa. En las últimas décadas la neurociencia, encargada de la organización y funcionamiento del sistema nervioso, ha emergido como una nueva disciplina para entender todos los enigmas que encierra el aprendizaje.

El cerebro posee una capacidad de aprehender y adaptarse a miles de entornos; registra todos los acontecimientos de nuestra vida hasta sus más ínfimos detalles; es el mago más poderoso que pueda existir jamás. Los neurocientíficos han descubierto que el sistema de conexión del cerebro es sorprendentemente plástico, lo que significa que puede ser modificado, y reconectarse según el tipo de experiencia que reciba. Las neuronas que descubrió el inolvidable científico español, Santiago Ramón y Cajal, juegan un papel prioritario en el aprendizaje creativo, pues ellas se ocupan de recibir y conducir los impulsos nerviosos, y se relacionan entre sí según sus funciones, formando infinidad de estructuras y redes neuronales.

Las neuronas, la materia prima del aprendizaje, interactúan entre ellas constantemente, creando redes neuronales o circuitos cerebrales, mediante un proceso que se llama sinapsis, que pueden ser de dos tipos: sinapsis eléctricas, un estímulo pasa de una célula a la siguiente, sin necesidad de mediación química, y sinapsis químicas mediante la intervención de neurotransmisores, como la serotonina, dopamina y oxitocina, entre otras. El cerebro no retrata la realidad como si fuera una máquina de fotos, sino que otorga un significado a las imágenes, retiene lo más importante y descarta los detalles que no le interesa, tanto en forma consciente, como inconsciente. La actividad cerebral interpreta lo que mira.

El cerebro es increíblemente misterioso. Algunos anatomistas han calculado que la corteza cerebral humana media contiene alrededor de treinta mil millones de neuronas. Cada neurona es autónoma e independiente, sin embargo, conecta con otras en un número aproximado de 1000 a 10000 enlaces o sinapsis, a través de las cuales puede dar y recibir 
información (Mora, 2002, p. 110). Según estos datos, la corteza cerebral se acercaría a los treinta mil billones de sinapsis.

Es de suma importancia para la educación las neuronas espejo, son aquellas que participan activamente en el aprendizaje, la imitación y la vida social; fue descubierta en 1996, mientras se realizaba un experimento con macacos, un equipo de la Universidad de Parma (Italia). Se observó que estas células no solo se encendían cuando el animal ejecutaba ciertos movimientos, sino también se activaban en el cerebro de los que estaban observando. Estas células tienen una enorme importancia para el desarrollo de técnicas de neuroaprendizaje. Mantener el cerebro activo es esencial para el aprendizaje creativo, pues ello ayudará a ser el escultor de tu propia arquitectura cerebral. Recojo un mensaje de Rodolfo Llinás, citado por Braidot (2014), neurocientífico colombiano, de talla internacional que afirma:

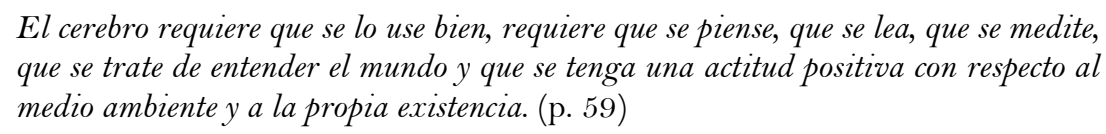

Por otra parte, el cerebro, morfológicamente, está constituido por dos hemisferios con funciones diferentes, unidos por el cuerpo calloso que hace de puente. El hemisferio izquierdo es el responsable de procesar la información de forma secuencial, verbal, lógica, lineal, temporal, analítica, convergente y se le reconoce funciones que tienen que ver con lo establecido. El hemisferio derecho trabaja en silencio, partiendo de presupuestos "puramente ficticios"; está especializado en la percepción global, holística, figurativa, espacial, asociativa, intuitiva, divergente y se le asigna las potencialidades creativas.

La creatividad resulta de la interacción entre ambos hemisferios y no el de uno solo, por más que la literatura siga identificándola con el hemisferio derecho. Se trata de una complementariedad de funciones (Mora, 2002, p. 52). El alumno tiene que aprender con todo el cerebro, valiéndose de los dos hemisferios, trabajando conjuntamente en equilibrio y armonía. La sinergia intrapersonal funciona cuando un alumno opera con la totalidad de su cerebro, es decir, tiene acceso al hemisferio izquierdo y también al hemisferio derecho.

El hipocampo, que tiene forma de caballito de mar, es una de las estructuras más relevantes, que desempeña un papel crucial en el aprendizaje, la formación de la memoria y el reconocimiento de los objetos. Sin un adecuado funcionamiento, el alumno no podrá recordar ni describir lo que ha visto, leído, escuchado o realizado pocos minutos antes. En el cerebro emocional o más conocido como sistema límbico se encuentra la amígdala, sustancia gris de forma almendrada, que se encarga de orquestar el mundo de las emociones, tan importante para el aprendizaje creativo; está conectada al neocórtex y al hipocampo. Aquí reside el amor y la felicidad, pero también el odio y la tristeza.

Una experiencia emocional puede ir de lo positivo y gratificante a lo negativo y punitivo. Los estímulos positivos generan sensaciones de felicidad y placer. Los estímulos emocionales negativos, que surgen cuando uno se enfada, generan una sensación de pérdida, y de miedo a sufrir un daño posible o real. Actualmente se ha incorporado en el mundo educativo un nuevo concepto: neuroaprendizaje, entendido como la capacidad del cerebro para percibir, incorporar y agrupar gran cantidad de información, en patrones neuronales y relacionarlos. Sabemos que los datos que entran en nuestro cerebro están teñidos por la percepción, el contexto emocional y la información almacenada en los sistemas de memoria. El neuroaprendizaje necesita de la percepción activa. Aprehender es convertir la información en conocimiento, útil para la vida, el crecimiento y desarrollo 
personal. Se trata de vivir y poner en práctica lo aprendido, con el objeto de transformar el conocimiento en sabiduría.

La capacidad para lograr cualquier meta radica en el poder interior que, tristemente, tenemos infrautilizado, incluso desactivado por completo. Hay que restaurar el funcionamiento de nuestro GPS interior y elevarlo a su máximo rendimiento. Un ambiente estimulante mantiene el cerebro en forma, igual que ocurre con la práctica regular del ejercicio físico. Una vida pasiva y adormecida es pobre en creatividad. Si el alumno aprende a desactivar los patrones antiguos y sabe activar su energía, estará en condiciones de reprogramar su cerebro y estimular la creatividad, desapareciendo las respuestas automáticas, es decir, los programas mentales de siempre. El desafío es dominar el concepto de aprehender y tener conciencia de los valores energéticos que determinan el aprendizaje creativo.

\section{El cerebro y el aprendizaje creativo}

\begin{abstract}
No olvides que el cerebro se desarrolla, expande su ramificación dendrítica y sus conexiones sinápticas cuando se mantienen alejadas las rutinas que llevan a automatismos cognitivos y conductuales.
\end{abstract}

(Braidot, 2014, p. 178)

¿Cómo se construye el aprendizaje creativo?

La construcción cerebral del aprendizaje ha sido investigada con todo detalle, por expertos de gran renombre internacional (Alonso Puig, 2013; Damasio, 1996, 2003, 2010; DelgadoGarcía, 2008; Mora, 2006, 2013; Ramón y Cajal, 2006; entre otros) quienes contemplan que distintas áreas del cerebro están implicadas en el proceso creativo.

El aprendizaje creativo crea nuevas redes neuronales y sincronías, que modifican el cerebro, proceso por el cual se conectan los conocimientos y se actualizan. El cerebro se expande cuando aumentan las conexiones sinápticas y se mantiene alejado de las rutinas, que llevan al automatismo cognitivo y conductual. Este fenómeno interactivo se ve influido no solo por cómo lo percibe el cerebro, sino también por los mapas mentales que se han ido construyendo, como resultado del aprendizaje y experiencias anteriores. Un cerebro habituado es un cerebro inactivo que envejece antes.

Cuando un alumno aprehende algo nuevo se establecen nuevas conexiones entre neuronas. $\mathrm{El}$ acto de aprehender genera nuevas dendritas y nuevas conexiones. Cuantas más ideas se atrapen más rico serán los circuitos neuronales. Si rememoramos esos recuerdos, aumentaremos la velocidad y la fuerza de las conexiones. La clave del aprendizaje creativo radica en la capacidad que posee el sistema nervioso para integrar estímulos de distinta naturaleza y generar una respuesta única. Cuando contemplamos un paisaje, no apreciamos formas, colores, tamaño, sonidos, y demás cualidades por separado, sino que todo se nos muestra de una percepción unitaria. La función integradora del sistema nervioso es imprescindible para captar la percepción.

El sistema nervioso autónomo opera por debajo del nivel de conciencia: El sistema simpático envía señales a los principales órganos preparándoles para el combate o la huida; el parasimpático es el responsable de la relajación. Estos dos sistemas están conectados de tal forma que cuando uno sube el otro baja. Para aprehender necesitamos ambos. 
A nivel cerebral, este proceso sensorial se desarrolla sin que seamos consciente y se caracteriza por etapas: ante cada estimulo captado por los sentidos, los receptores envían las señales al área correspondiente, después se dirigen al tálamo, y desde allí, al área propia de la corteza sensorial. Si el estímulo percibido evoca algún tipo de emoción participará el sistema límbico, dominado por la amígdala, como ya se ha indicado. Las técnicas de estimulación multisensorial son estrategias educativas que facilitan el aprendizaje creativo. Los neurotransmisores son las sustancias químicas que transmiten información, mediante la sinapsis, en la comunicación que se establece entre las neuronas. Hay sustancias químicas para cada estado emocional: para el amor, la alegría y la felicidad están la serotonina y la dopamina; así como para la tristeza, el odio y el enfado está el cortisol. Y cada vez que se activa el pensamiento (positivo o negativo), el hipotálamo, inmediatamente, libera esa hormona en la corriente sanguínea.

$\mathrm{El}$ conocimiento de estos mensajeros es fundamental para comprender el funcionamiento de los procesos cerebrales y optimizar el desarrollo del aprendizaje y la memoria. Por ejemplo, el glutamato participa en las funciones del aprendizaje y la memoria; la dopamina es esencial en la motivación, las emociones y el sentimiento de placer y la serotonina regula el estado anímico, el sueño y el humor. El impacto del estrés, las preocupaciones y el miedo provocan en los alumnos fuertes estados de angustia psicológica y ansiedad que se traducen en un malestar emocional y físico, lo que reduce la actividad de las funciones ejecutivas del cerebro y provocan dificultades en el aprendizaje, atención, concentración, memoria y toma de decisiones.

Todo ser humano nace, crece, aprehende y actúa en un entorno con el cual intercambia energía e información. Durante este proceso, las sensaciones que experimenta son el resultado de la interacción de millones de neuronas que envían y reciben mensajes por una enorme cantidad de redes neuronales interconectadas. Cada sujeto interpreta el mundo en función de lo que le interesa. Todos los procesos del cerebro son complejos, extraordinarios y fascinantes a la vez. Para aprehender de forma óptima se recomienda utilizar todos los sentidos y una variedad de actividades, durante el tiempo que dure el proceso. Cuantas más regiones del cerebro se activen, mayor será la capacidad de procesar, sinergizar y desarrollar nuevos conocimientos. Sabemos por la escuela psicológica de la Gestalt que, el sujeto humano percibe totalidades, no meros agregados de elementos constituyentes.

$\mathrm{El}$ arte de prestar atención, requiere una fuerza de voluntad consciente; es imprescindible para una adecuada percepción. Lo que sucede, en realidad, es que el aprendiz no observa de forma concentrada, porque está interesado en otras cosas, que absorbe la mente. Esta es una de las causas por la que el alumno no aprende, mira, pero no ve, debido a que su foco de atención está dirigido hacia algo específico (preocupación, angustia, enfado, soledad, miedo). La percepción es inviable sin la atención. Para que el aprendizaje sea creativo es necesario concentrarse en la tarea durante un tiempo prolongado, activando así los circuitos de la atención sostenida, que luego se traducirá en una mayor facilidad en recordar y memorizar datos o hechos. Cuando se es capaz de reflexionar sobre lo que ocurre, se está en condiciones de abrir la puerta para acceder al mundo que le rodea.

Por lo cual, hay que considerar que el mecanismo cerebral más importantes para realizar el aprendizaje creativo es estimular la capacidad de percibir la realidad, sean hechos, experiencias, conceptos o situaciones. Todo ello implica activar un conjunto de procesos neurobiológicos que permitan comprender el entorno escolar en el que se está, otorgar 
significado a lo que ocurre y actuar de una determinada manera. Los docentes, para desarrollar un programa de aprendizaje concreto, deberían aplicar el poder del denominado efecto placebo, igual que hacen los médicos con su paciente y los deportistas $\mathrm{y}$ artistas lo emplean para mejorar sus habilidades. El docente debe enseñar y entrenar al alumno a que es posible lograr todo lo que se proponga, siempre y cuando sepa concentrar sus pensamientos en el aprendizaje que quiere alcanzar y visualizar emocionalmente, con todo tipo de detalle, que ya lo ha conseguido. Lo único que hace falta es voluntad y decisión para atraer a los pensamientos positivos Los individuos tienen el cerebro ralentizado.

\section{A modo de conclusión}

Cada generación hereda un impulso interior de desarrollo a partir de la generación anterior.

(Chopra, 2006, p. 150)

¿Qué ocurre en el interior del cerebro cuando estamos aprendiendo?

Los niños de hoy, que están sentados en nuestras aulas, pronto estarán configurando el mundo y dirigiendo nuestra sociedad; es nuestra responsabilidad darles una educación holística, de calidad y creativa, que despierte el interés por saber, y alejadas de las cargas de la educación del siglo XIX que todavía se arrastran.

El reto de la educación en las próximas décadas no será, simplemente, el dominio del conocimiento, sino el poder del aprendizaje creativo. Por esta razón, los docentes deberán implicarse y comprometerse con el nuevo paradigma; o dicho de otra forma, los estudiantes que practican el aprendizaje repetitivo, han de transformarse y evolucionar hacia el aprendizaje creativo, quienes disfrutarán de poder elegir qué aprehender, cómo aprehender, teniendo la oportunidad de seleccionar múltiples maneras de hacerlo, según sus circunstancias. La preocupación central de los docentes será potenciar el aprendizaje creativo, y así ampliar las posibilidades para empoderarse. Stiglitz y Greenwald (2016), premio Nobel de Economía en 2001, habla de la creación de una sociedad del aprendizaje.

Los alumnos del futuro tienen que aprehender de forma permanente y creativa, y tienen que hacerlo mejor que aquellos que vivieron en la era industrial, igual que éstos lo hicieron de forma distinta a los que pertenecieron a la sociedad agrícola. El estudiante que sale con su teléfono móvil y se comunica por WhatsApp, no puede aprehender igual que aquel otro que en su tiempo libre jugaba con el trompo. Tienen diferencias notables en las posibilidades cognitivas de sus cerebros. Los centros educativos deben promocionar la cultura del aprendizaje creativo, caracterizada por el poder transformador. Siendo nuestra intención impulsar a todos los docentes hacia un proyecto de transformación, cristalizado en la implantación del aprendizaje creativo en el aula, consideramos clave el cambio de mirada de los docentes, como exponemos a continuación:

Cada alumno tiene su ritmo particular de aprendizaje creativo que debemos respetar: Descubrir la forma de aprehender es la primera tarea didáctica que debe realizar el docente y después, ha de diseñar el camino a recorrer, sorteando las limitaciones y alcanzando, felizmente, cada momento de la evolución. Lo que importa es la búsqueda, no el objetivo. Las expectativas que se crean influyen decisivamente sobre el aprendizaje creativo, así como la atención e intención empleado en el proceso. Es urgente repensar cómo enseñar, cómo aprehender y cómo crear contextos autotélicos, para que los alumnos, que son nativos digitales, saquen provecho a su experiencia de aprendizaje. Cada alumno debe 
construir su propio aprendizaje en función de la lupa de su propia percepción, Su responsabilidad será adquirir el compromiso y sentirse implicado y motivado por conectarse con su proyecto vital.

Para que el aprendizaje creativo sea efectivo se requiere que el proceso cerebral empleado funcione con armonía: En el proceso de aprendizaje creativo la autoestima y la confianza es determinante; un alumno comprometido hace que las funciones cerebrales se canalicen hacia el éxito. Si los pensamientos son negativos, configurarán neurocircuitos que, inevitablemente, conducirán al fracaso. El pensamiento dominante crea realidades y tiene tanto poder para atraer felicidad y éxito, como para atraer tristeza y fracaso. El docente debe tener presente que los contenidos del cerebro lo constituyen tres campos: las formas mentales, donde reposa el conocimiento, las formas afectivas, donde se encuentran los sentimientos y emociones y la forma energética, donde la voluntad puede activar las potencialidades. Todos estos contenidos están en un fluir constante de renovación e intercambio. Es necesario estimular los tres campos para que el aprendizaje creativo pueda florecer.

El gran reto del futuro será dinamizar el cuerpo, dosificar la mente y divinizar el alma, única forma de que florezca el magnetismo que el niño trae en su interior. Este torrente de energía que proyecta la naturaleza humana no puede mantenerse en la oscuridad. Para evolucionar es imprescindible que surja el aprendizaje creativo a partir de la acción de aprehender. La escuela e institutos necesitan que los docentes promuevan mejores experiencias, mejores prácticas y mejores talleres.

Reivindicamos el aprendizaje creativo de los alumnos a todos los niveles, desde que se pisa un aula por primera vez, hasta los estudios universitarios. La revolución creativa es responsabilidad de todos. Para que el docente gestione el cambio que proponemos es necesario salirse de la zona de confort e inspirar a los alumnos a desarrollar habilidades nuevas y despertar pasión por aprehender.

\section{Referencias}

Alonso Puig, M. (2013). El cociente agalla. Sé valiente, cambia tu vida. Barcelona: Espasa.

Braidot, N. (2014). Cómo funciona tu cerebro. Barcelona: Planeta.

Ramón y Cajal, S. (2006). Recuerdos de mi vida. Barcelona: Crítica.

Chopra, D. (2006). Energía sin límites. Barcelona: Ediciones B.

Damasio, A. (1996). El error de Descartes. La emoción, la razón y el cerebro humano. Barcelona: Crítica.

Damasio, A. (2003). En busca de Spinoza. Neurobiología de la emoción y los sentidos. Barcelona: Crítica.

Damasio, A. (2010). Y el cerebro creó al hombre. ¿Cómo puede el cerebro generar emociones, sentimientos, ideas y el yo? Barcelona: Destino.

Delgado-García, J. M. (2008). El lenguaje del cerebro. Sevilla: Letra Aurea.

Mora, F. (2002). Cómo funciona el cerebro. Madrid: Alianza Editorial.

Mora, F. (2006). Los laberintos del placer en el cerebro. Madrid: Alianza.

Mora, F. (2013). Neuroeducación. Solo se puede aprender aquello que se ama. Madrid: Alianza Editorial.

Saint Aymour, J. (2015). Conexión cuántica. Claves para convertir lo imaginado en realidad. Cómo sincronizar con todo lo que deseas. Barcelona: Obelisco. 
Stiglitz, J. E. y Greenwald, B. C. (2016). La creación de una sociedad del aprendizaje. Madrid: Esfera de los libros.

Torrance, P. (1976). La energía creativa. Madrid: Santillana.

Wilber, K. (2016). Meditación integral. Mindfulness para despertar y estar presente en nuestra vida. Barcelona: Kairós.

\section{Breve CV de autor}

\section{Francisco Menchén Bellón}

Licenciado en Psicología y en Ciencias de la Educación. Ha sido Inspector de Educación en la Comunidad de Madrid. Actualmente, Explorador y “Curator” de la Creatividad. Cuenta con varios Premios Nacionales de Investigación. Ha dictado conferencias en España, Portugal, México, Brasil, Perú, Colombia y El Salvador. Cuenta con más de una docena de libros relacionados con el mundo educativo. Hace veinte años escribió: Descubrir la Creatividad. Desaprender para volver aprender. En 2015 ha publicado La necesidad de Escuelas Creativas. La Escuela Galáctica. Una Nueva Conciencia. Próximamente se editará Cómo capacitar excelentes docentes innovadores. Sistema Creativo del ser humano. ORCID ID: https://orcid.org/OOOO-0002-0034-6373. Email: fcomenchen@yahoo.com 